\title{
COEFFICIENT ESTIMATES FOR DIRICHLET SERIES
}

\author{
BY \\ W. T. SLEDD $\left({ }^{1}\right)$
}

1. Introduction. The primary purpose of this paper is to study coefficient estimates of Dirichlet series

$$
f(x)=\sum_{n=1}^{\infty} a_{n} \exp \left(-\lambda_{n} x\right), \quad \lambda_{n} \rightarrow \infty, \quad 0<\lambda_{1}<\lambda_{2}<\cdots,
$$

for which the sequence $\left\{\lambda_{n}\right\}$ satisfies certain lacunarity conditions. D. Gaier [2] has used such estimates in one of his proofs of the high-indices theorem for Borel summability, in which results on the order of an entire function are inferred from its rate of growth along the real line. A by-product of his work is the estimate $\left|a_{n}\right| \leqq 2 \lambda_{n} p_{n}\|f\|_{1}$ when $\sum \lambda_{n}^{-1}<\infty, f \in L^{1}(0, \infty)$, and

$$
p_{n}=\prod_{k \neq n}\left|\frac{\lambda_{k}+\lambda_{n}}{\lambda_{k}-\lambda_{n}}\right| \text {. }
$$

Some subsequent papers in much the same vein are due to G. Halász [3] and to J. M. Anderson and K. G. Binmore [1]. Halász has obtained estimates for $a_{n}, \sum_{1}^{N} a_{n}$, and $\sum_{1}^{N}\left|a_{n}\right|$ when $\lambda_{n+1} / \lambda_{n} \geqq q>1$ and $f$ satisfies appropriate conditions. The paper of Anderson and Binmore is concerned with the application of coefficient estimates to the study of entire functions. Their work includes the estimate $\left|a_{n}\right|$ $\leqq\left(2 \lambda_{n}\right)^{1 / 2} p_{n}\|f\|_{2}$ where $p_{n}$ is as above, $\sum \lambda_{n}^{-1}<\infty$, and $f \in L^{2}(0, \infty)$.

L. Schwartz [8] had earlier obtained results which essentially include both the estimates of Gaier and of Anderson and Binmore. Indeed, as the referee has pointed out, S. Mandelbrojt [5] had also established inequalities of the same kind and these appear in larger form in [6]. Later, Mandelbrojt's results were extended by F. Sunyer Belaguer [9], [10].

There is some question left to the sharpness of Schwartz's estimates and in $\$ 2$ it is proved that if $1 \leqq p \leqq 2$ and $f \in L^{p}(0, \infty)$ then

$$
\left|a_{n}\right| \leqq\left(2 \lambda_{n}\right)^{1 / p} p_{n}\|f\|_{p} .
$$

I do not know if the constant is sharp when $p<2$, or if the order of the estimate still holds when $p>2$ but in $\S 2$ there are estimates when $p>2$ for functions which are subject to more stringent conditions. Also in $\$ 2$ there are estimates for the sequence of partial sums.

Received by the editors February 18, 1969.

(1) Research sponsored by NSF Grant GP-7840.

Copyright $(1)$ 1970, American Mathematical Society 
These results are applied in $\$ 3$ to obtain some gap theorems. W. Rudin [7] showed that if lim inf $\lambda_{n+1} / \lambda_{n}=1$ then there is a Dirichlet series so that $f^{\prime} \in L^{1}(0, \infty)$ yet $\sum a_{n}$ diverges. In $\S 3$ it is shown that there are sequences $\left\{\lambda_{n}\right\}$ so that $\lim \lambda_{n+1} / \lambda_{n}$ $=1$, but so that if $f^{\prime} \in L^{p}(0, \infty)$ when $p>1$, or if $f^{\prime \prime} \in L^{1}(0, \infty)$, then $\sum\left|a_{n}\right|<\infty$.

\section{Coefficient estimates.}

THEOREM 2.1. If $f(x)=\sum_{n=1}^{\infty} a_{n} \exp \left(-\lambda_{n} x\right)$ converges for each $x>0, \sum \lambda_{n}^{-1}<\infty$, and $f \in L^{p}(0, \infty), 1 \leqq p \leqq 2$, then $\left|a_{n}\right| \leqq\left(2 \lambda_{n}\right)^{1 / p} p_{n}\|f\|_{p}$ where

$$
p_{n}=\prod_{k \neq n}\left|\frac{\lambda_{k}+\lambda_{n}}{\lambda_{k}-\lambda_{n}}\right| \text {. }
$$

Proof. Let

$$
f_{N}(x)=\sum_{k=1}^{N} a_{k} \exp \left(-\lambda_{k} x\right)
$$

and

$$
F(z)=\sum_{k=1}^{N} \frac{a_{k} \exp \left(-\lambda_{k} \varepsilon\right)}{\lambda_{k}-z}, \quad \varepsilon>0
$$

Then if $\operatorname{Re} z<0$

$$
F(z)=\int_{0}^{\infty} e^{z t} f_{N}(t+\varepsilon) d t
$$

Let

$$
B(z)=\prod_{k=1}^{N} \frac{\lambda_{k}-z}{\lambda_{k}+z}
$$

so that

$$
G(z)=F(z) B(z)=\sum_{k=1}^{N} \frac{a_{k} \exp \left(-\lambda_{k} \varepsilon\right)}{z+\lambda_{k}} B_{k}(z)
$$

where

$$
B_{k}(z)=\left(\prod_{j \neq k}^{N} \frac{\lambda_{j}-z}{\lambda_{j}+z}\right)
$$

Now $B_{k}(z) /\left(z+\lambda_{k}\right) \in H^{2}$, a Hardy class of functions in the right half-plane, and so the same is true of $G$. Consequently, $G$ may be represented by the Poisson integral of its values on the imaginary axis, and

$$
\|G(x+i y)\|_{q} \leqq\|G(i y)\|_{q}
$$

if $x>0$ and $q \geqq 1$ [4, p. 124, and p. 128].

Next, let $\Gamma$ be the boundary of a rectangle in the right half-plane with sides parallel to the real and imaginary axes, and enclosing $\lambda_{n}$.

Let $\zeta^{2 / p}=\exp (2 / p \log \zeta)$ for $|\arg \zeta|<\pi / 2$, with $\log 1=0$. Then by the residue theorem

$$
\frac{a_{n} \exp \left(-\lambda_{n} \varepsilon\right) B_{n}\left(\lambda_{n}\right)}{\left(2 \lambda_{n}\right)^{2 / p}}=\frac{-1}{2 \pi i} \int_{\Gamma} \frac{F(z) B_{n}(z)}{\left(z+\lambda_{n}\right)^{2 / p}} d z
$$


But since $G(z)$ goes to zero uniformly in any fixed half-plane $\operatorname{Re} z \geqq \delta>0$ [4, p. 125] it follows that

$$
\begin{aligned}
\frac{a_{n} \exp \left(-\lambda_{n} \varepsilon\right) B_{n}\left(\lambda_{n}\right)}{\left(2 \lambda_{n}\right)^{2 / p}}= & \frac{1}{2 \pi} \int_{-\infty}^{\infty} \frac{F\left(x_{n}^{\prime}+i y\right) B_{n}\left(x_{n}^{\prime}+i y\right)}{\left(x_{n}^{\prime}+i y+\lambda_{n}\right)^{2 / p}} d y \\
& -\frac{1}{2 \pi} \int_{-\infty}^{\infty} \frac{F\left(x_{n}^{\prime \prime}+i y\right) B_{n}\left(x_{n}^{\prime \prime}+i y\right)}{\left(x_{n}^{\prime \prime}+i y+\lambda_{n}\right)^{2 / p}} d y \\
= & I_{1}+I_{2}
\end{aligned}
$$

where $x_{n}^{\prime}<\lambda_{n}<x_{n}^{\prime \prime}$.

Then

$$
\left|I_{1}\right| \leqq \frac{1}{2 \pi}\left\|G\left(x_{n}^{\prime}+i y\right)\right\|_{q}\left[\int_{-\infty}^{\infty}\left|\frac{x_{n}^{\prime}+i y+\lambda_{n}}{x_{n}^{\prime}+i y-\lambda_{n}}\right|^{p} \frac{d y}{\left|x_{n}^{\prime}+i y+\lambda_{n}\right|^{2}}\right]^{1 / p}
$$

where $1 / p+1 / q=1$. A similar estimate holds for $I_{2}$. Since $\left\|G\left(x_{n}^{\prime \prime}+i y\right)\right\|_{q} \leqq\|G(i y)\|_{q}$ we may let $x_{n}^{\prime \prime} \rightarrow \infty$ and $x_{n}^{\prime} \rightarrow 0$ to conclude that

$$
\begin{aligned}
\frac{\left|B_{n}\left(\lambda_{n}\right) a_{n} \exp \left(-\lambda_{n} \varepsilon\right)\right|}{\left(2 \lambda_{n}\right)^{2 / p}} & \leqq \frac{1}{2 \pi}\|G(i y)\|_{q}\left(\int_{-\infty}^{\infty} \frac{d y}{y^{2}+\lambda_{n}^{2}}\right)^{1 / p} \\
& =\frac{1}{2 \pi}\left(\frac{\pi}{\lambda_{n}}\right)^{1 / p}\|G(i y)\|_{q} .
\end{aligned}
$$

Moreover, since $|B(i y)|=1$, then $\|G(i y)\|_{q}=\|F(i y)\|_{q}$, but $F(i y)$ is the Fourier transform of $f_{N}(t+\varepsilon)$, so that by the Hausdorff-Young inequality [11, p. 96], $\|G(i y)\|_{q}$ $\leqq(2 \pi)^{1 / q}\left\|f_{N}(t+\varepsilon)\right\|_{p}$.

Combining these inequalities gives

$$
\left|a_{n} \exp \left(-\lambda_{n} \varepsilon\right)\right| \leqq \frac{\left(2 \lambda_{n}\right)^{1 / p}}{\left|B_{n}\left(\lambda_{n}\right)\right|}\left\|f_{N}(t+\varepsilon)\right\|_{p}
$$

If $\varepsilon>0$, summation by parts shows that there is a constant $C$ such that

$$
\left|f_{N}(t+\varepsilon)\right| \leqq C \exp \left(-\lambda_{1} t\right)
$$

and

$$
|f(t+\varepsilon)| \leqq C \exp \left(-\lambda_{1} t\right) .
$$

Since $f_{N}(t) \rightarrow f(t)$ when $t>0$, the Lebesgue dominated convergence theorem implies that

$$
\left\|f_{N}(t+\varepsilon)-f(t+\varepsilon)\right\|_{p} \rightarrow 0 .
$$

But $\|f(t+\varepsilon)\|_{p} \leqq\|f\|_{p}$ and so letting $N \rightarrow \infty$ and then $\varepsilon \rightarrow 0$ in the above inequality completes the proof.

The next theorem deals with the case where $p>2$. The technique is essentially that of Halász [3]. 
THEOREM 2.2. If

(a) $f(z)=\sum_{n=1}^{\infty} a_{n} \exp \left(-\lambda_{n} z\right)$ converges when $\operatorname{Re} z>0$;

(b) $\left\|f\left(r e^{i \theta}\right)\right\|_{p} \leqq M$ whenever $|\theta| \leqq \eta=\delta \pi / 2<\pi / 2$;

(c) $\sum \lambda_{n}^{-1 / \beta}<\infty$, where $\beta+\delta=\gamma>1$, and $\beta<2$; then

where $1 / p+1 / q=1$.

$$
\left|a_{n}\right| \leqq \frac{2 M \beta \lambda_{n}^{1 / p}}{|q \cos (\gamma \pi / 2)|^{1 / q}} \prod_{k \neq n}\left|\frac{\lambda_{k}^{1 / \beta}+\lambda_{n}^{1 / \beta}}{\lambda_{k}^{1 / \beta}-\lambda_{n}^{1 / \beta}}\right|
$$

Proof. Let

$$
\phi_{N}(z)=\sum_{k=1}^{N} a_{k} \exp \left(-\lambda_{k} z\right)
$$

and

$$
F(z)=\sum_{k=1}^{N} \frac{a_{k} \exp \left(-\lambda_{k} \varepsilon\right)}{\lambda_{k}-z}, \quad \varepsilon>0 .
$$

Then if $\operatorname{Re} z e^{i \alpha}<0$, and $|\alpha|<\pi / 2$

$$
F(z)=\int_{0}^{\infty} \exp \left(z t e^{i \alpha}\right) \phi_{n}\left(t e^{i \alpha}+\varepsilon\right) e^{i \alpha} d t .
$$

But if $z=r e^{i \phi}$ and $\operatorname{Re} z e^{i \alpha}<0$, then

$$
\begin{aligned}
|F(z)| & \leqq\left[\int_{0}^{\infty} \exp (r t q \cos (\theta+\alpha)) d t\right]^{1 / q}\left\|\phi_{N}\left(t e^{i \alpha}+\varepsilon\right)\right\|_{p} \\
& =\left\|\phi_{N}\left(t e^{i \alpha}+\varepsilon\right)\right\|_{p} \frac{1}{|q r \cos (\theta+\alpha)|^{1 / q}} .
\end{aligned}
$$

Now let $(\zeta)^{1 / \beta}$ be defined for $|\arg \zeta|<\pi,(1)^{1 / \beta}=1$, and set

$$
B(z)=\prod_{1}^{N} \frac{1-\left(z / \lambda_{k}\right)^{1 / \beta}}{1+\left(z / \lambda_{k}\right)^{1 / \beta}}
$$

Similarly define $(\zeta)^{1 / q}$, so that letting

$$
\begin{gathered}
\alpha= \pm \eta, \quad z=r \exp ( \pm i \pi \beta / 2) \\
\left|z^{1 / q} F(z) B(z)\right| \leqq \frac{\left\|\phi_{N}\left(t e^{i \alpha}+\varepsilon\right)\right\|_{p}}{|q \cos (\gamma \pi / 2)|^{1 / q}}=A
\end{gathered}
$$

Now $z^{1 / q} F(z) B(z)$ is of relatively slow growth for $|\arg z|<\beta \pi / 2$, so that by the Phrägmen-Lindelöf theorems [12, p. 180]

$$
\left|z^{1 / q} F(z) B(z)\right| \leqq A, \quad|\arg z| \leqq \beta \pi / 2 .
$$

In particular, if $z=\lambda_{n}$ then

$$
\frac{\lambda_{n}^{1 / q}\left|a_{n}\right| \exp \left(-\lambda_{n} \varepsilon\right)}{2 \beta \lambda_{n}} \prod_{k \neq n}\left|\frac{\lambda_{k}^{1 / \beta}-\lambda_{n}^{1 / \beta}}{\lambda_{k}^{1 / \beta}+\lambda_{n}^{1 / \beta}}\right| \leqq A .
$$

As in the proof of Theorem 2.1, let $N \rightarrow \infty$ and then $\varepsilon \rightarrow 0$ to obtain the desired inequality. 
The next theorem deals with sequences of partial sums.

THEOREM 2.3. If $f(x)=\sum_{n=1}^{\infty} a_{n} \exp \left(-\lambda_{n} x\right)$ converges for each $x>0, \sum \lambda_{n}^{-1}<\infty$ and $f^{\prime} \in L^{p}(0, \infty), 1<p \leqq 2$, then

$$
\left|\sum_{k=r}^{s} a_{k}\right| \leqq A_{p}\left\|f^{\prime}\right\|_{p}\left[C_{r-1}+C_{s}\right]
$$

where $A_{p}$ is a constant depending on $p$ alone, and

$$
C_{n}=\operatorname{Min}_{\lambda_{n}<x<\lambda_{n+1}} \frac{1}{x^{1-1 / p}} \prod_{k=1}^{\infty}\left|\frac{x+\lambda_{k}}{x-\lambda_{k}}\right| .
$$

Proof. The proof is very much the same as that of Theorem 2.1 and hence many of the details are omitted.

Let

$$
\begin{gathered}
\phi_{N}(x)=\sum_{k=1}^{N} \lambda_{k} a_{k} \exp \left(-\lambda_{k} x\right), \\
F(z)=\sum_{k=1}^{N} \frac{\lambda_{k} a_{k} \exp \left(-\lambda_{k}(z+\varepsilon)\right)}{\lambda_{k}-z}, \quad \varepsilon>0,
\end{gathered}
$$

and

$$
B(z)=\prod_{k=1}^{N} \frac{\lambda_{k}-z}{\lambda_{k}+z}
$$

If $\Gamma$ is a rectangle in the right half-plane enclosing $\lambda_{r}, \ldots, \lambda_{s}$ and no others then

$$
\begin{aligned}
\sum_{k=r}^{s} a_{k} \exp \left(-\lambda_{k} \varepsilon\right) & =-\frac{1}{2 \pi i} \int \frac{F(z)}{z} d z \\
& =\frac{1}{2 \pi} \int_{-\infty}^{\infty} \frac{F\left(x_{r}+i y\right)}{x_{r}+i y} d y-\frac{1}{2 \pi} \int_{-\infty}^{\infty} \frac{F\left(x_{s}+i y\right)}{x_{s}+i y} d y \\
& =I_{1}+I_{2},
\end{aligned}
$$

where $\lambda_{r-1}<x_{r}<\lambda_{r} \leqq \lambda_{s}<x_{s}<\lambda_{s+1}$.

If $G(z)=F(z) B(z)$ then

$$
\left|I_{1}\right| \leqq \frac{1}{2 \pi}\left\|G\left(x_{r}+i y\right)\right\|_{q} D_{r}\left(\int_{-\infty}^{\infty} \frac{d y}{\left|x_{r}+i y\right|^{p}}\right)^{1 / p}
$$

where $1 / p+1 / q=1$ and

$$
D_{r}=\max _{y} \frac{1}{\left|B\left(x_{r}+i y\right)\right|}
$$

A similar estimate holds for $I_{2}$ and since

$$
\left\|G\left(x_{r}+i y\right)\right\|_{q} \leqq\|G(i y)\|_{q}=\|F(i y)\|_{q} \leqq(2 \pi)^{1 / q}\left\|\phi_{N}(t+\varepsilon)\right\|_{p}
$$

it follows that

$$
\left|\sum_{k=r}^{s} a_{k} \exp \left(-\lambda_{k} \varepsilon\right)\right| \leqq A_{p}\left\|\phi_{N}(t+\varepsilon)\right\|_{p}\left(\frac{D_{r}}{x_{r}^{q}}+\frac{D_{s}}{x_{s}^{q}}\right) .
$$

Letting $N \rightarrow \infty$ and then $\varepsilon \rightarrow 0$ completes the proof. 
THEOREM 2.4. If $f(x)=\sum_{n=1}^{\infty} a_{n} \exp \left(-\lambda_{n} x\right)$ converges for each $x>0, \sum \lambda_{n}^{-1}<\infty$, and $f^{\prime \prime} \in L^{1}(0, \infty)$ then

$$
\left|\sum_{k=r}^{s} a_{k}\right| \leqq \frac{\left\|f^{\prime \prime}\right\|}{2}\left[\operatorname{Min}_{\lambda_{r}-1<x<\lambda_{r}} \frac{1}{x} \prod_{k=1}^{\infty}\left|\frac{x+\lambda_{k}}{x-\lambda_{k}}\right|+\underset{\lambda_{s}<x<\lambda_{s+1}}{\operatorname{Min}} \frac{1}{x} \prod_{k=1}^{\infty}\left|\frac{x+\lambda_{k}}{x-\lambda_{k}}\right|\right] .
$$

Proof. The proof is very similar to the proof of Theorem 2.3 and so will be omitted.

3. Some applications. Before applying the estimates of $\S 2$ it is necessary to establish a result about the special sequence $\lambda_{n}=\exp \left(n^{\alpha}\right)$.

THEOREM 3.1. If $\lambda_{n}=\exp \left(n^{\alpha}\right)$ and $1>\alpha>0$ then

$$
P_{n}=\prod_{k \neq n}\left|\frac{\lambda_{k}+\lambda_{n}}{\lambda_{k}-\lambda_{n}}\right| \leqq \exp C n^{2(1-\alpha)}
$$

where $C$ is a constant, dependent only on $\alpha$.

Proof. Since $\lambda_{k} / \lambda_{k-1} \geqq \lambda_{k+1} / \lambda_{k}$, it follows that

$$
\begin{aligned}
\sum_{k=1}^{n-1} \frac{\lambda_{k}}{\lambda_{n}-\lambda_{k}} & =\sum_{k=1}^{n-1} \frac{\lambda_{k}}{\lambda_{n}} \sum_{j=0}^{\infty}\left(\frac{\lambda_{k}}{\lambda_{n}}\right)^{j}=\sum_{j=1}^{\infty} \frac{1}{\lambda_{n}^{j}} \sum_{k=1}^{n-1} \lambda_{k}^{j} \\
& \leqq \sum_{j=1}^{\infty} \frac{1}{\lambda_{n}^{j}} \lambda_{n-1}^{j-1} \sum_{k=1}^{n-1} \lambda_{k} \leqq \sum_{j=1}^{\infty}\left(\frac{\lambda_{n-1}}{\lambda_{n}}\right)^{j} \frac{1}{1-\lambda_{n-2} / \lambda_{n-1}} \\
& \leqq \frac{1}{1-\lambda_{n-1} / \lambda_{n}} \frac{1}{1-\lambda_{n-2} / \lambda_{n-1}} \sim \frac{1}{\alpha^{2} n^{2(\alpha-1)}} .
\end{aligned}
$$

And

But

$$
\sum_{k=n+1}^{\infty} \frac{\lambda_{n}}{\lambda_{k}-\lambda_{n}}=\sum_{j=1}^{\infty} \lambda_{n}^{j} \sum_{k=n+1}^{\infty} \frac{1}{\lambda_{k}^{j}}
$$

so that

$$
\sum_{k=n+1}^{\infty} \frac{1}{\lambda_{k}^{j}} \leqq \frac{1}{\lambda_{n+1}^{j}}+\int_{n+1}^{\infty} \exp \left(-j x^{\alpha}\right) d x
$$

$$
\begin{aligned}
\sum_{j=1}^{\infty} \lambda_{n}^{j} \sum_{k=n+1}^{\infty} \frac{1}{\lambda_{k}^{j}} & \leqq \sum_{j=1}^{\infty}\left(\frac{\lambda_{n}}{\lambda_{n+1}}\right)^{j}+\int_{n+1}^{\infty} \sum_{j=1}^{\infty}\left(\frac{\lambda_{n}}{\exp \left(x^{\alpha}\right)}\right)^{j} d x \\
& \leqq \frac{\lambda_{n}}{\lambda_{n+1}} \frac{1}{1-\lambda_{n} / \lambda_{n+1}}+\frac{1}{\alpha} \int_{(n+1)^{\alpha}}^{\infty} \frac{\lambda_{n} e^{-u}}{u^{1-1 / \alpha}} \frac{d u}{1-\lambda_{n} e^{-u}} \\
& \leqq \frac{\lambda_{n}}{\lambda_{n+1}} \frac{1}{1-\lambda_{n} / \lambda_{n+1}}+\frac{1}{\alpha} \frac{\lambda_{n}}{1-\lambda_{n} / \lambda_{n+1}} \int_{(n+1)^{\alpha}}^{\infty} u^{1 / \alpha-1} e^{-u} d u .
\end{aligned}
$$

But

$$
\begin{aligned}
\int_{(n+1)^{\alpha}}^{\infty} u^{1 / \alpha-1} e^{-u} d u & =(n+1)^{1-\alpha} \lambda_{n+1}^{-1}+\frac{1-\alpha}{\alpha} \int_{(n+1)^{\alpha}}^{\infty} \frac{e^{-u} u^{1 / \alpha-1}}{u} d u \\
& \leqq(n+1)^{1-\alpha} \lambda_{n+1}^{-1}+\frac{1-\alpha}{\alpha} \frac{1}{(n+1)^{\alpha}} \int_{(n+1)^{\alpha}}^{\infty} e^{-u} u^{1 / \alpha-1} d u,
\end{aligned}
$$


so for large values of $n$

$$
\begin{aligned}
\sum_{k=n+1}^{\infty} \frac{1}{\lambda_{k}^{j}} & \leqq \frac{1}{1-\lambda_{n} / \lambda_{n+1}}+\frac{1}{\alpha} \frac{(n+1)^{1-\alpha}}{1-\lambda_{n} / \lambda_{n+1}}\left(1-\left(\frac{1-\alpha}{\alpha(n+1)^{\alpha}}\right)\right)^{-1} \\
& \sim \frac{1}{\alpha n^{\alpha-1}}+\frac{1}{\alpha^{2}} \frac{1}{(n+1)^{2(\alpha-1)}} \leqq \frac{2}{\alpha^{2}} \frac{1}{(n+1)^{2(\alpha-1)}}
\end{aligned}
$$

So

$$
\begin{aligned}
p_{n} & =\prod_{k=1}^{n-1} \frac{\lambda_{k}+\lambda_{n}}{\lambda_{n}-\lambda_{k}} \prod_{k=n+1}^{\infty} \frac{\lambda_{k}+\lambda_{n}}{\lambda_{k}-\lambda_{n}} \\
& \leqq \exp 2 \sum_{k=1}^{n-1} \frac{\lambda_{k}}{\lambda_{n}-\lambda_{k}} \cdot \exp 2 \sum_{k=n+1}^{\infty} \frac{\lambda_{n}}{\lambda_{k}-\lambda_{n}} \\
& \leqq \exp C n^{2(1-\alpha)}
\end{aligned}
$$

where $C$ is some constant.

With this estimate the following theorems may be proved.

THEOREM 3.2. If $p>1$ then there is a sequence $\left\{\lambda_{n}\right\}$ such that if

$$
f(x)=\sum_{n=1}^{\infty} a_{n} \exp \left(-\lambda_{n} x\right)
$$

converges for each $x>0$ and $f^{\prime} \in L^{p}(0, \infty)$ then $\sum\left|a_{n}\right|^{\varepsilon}<\infty$ for each $\varepsilon>0$.

Proof. Assume without loss of generality that $1<p \leqq 2$, for if $p>2$ and $f^{\prime} \in L^{p}(0, \infty)$ then $f^{\prime} \in L^{r}(0, \infty)$ for each $r<p$. This is true since the integrability of $f^{\prime}$ depends only on its integrability over $(0,1)$.

Now by Theorem 2.1 and Theorem 3.1, if $\lambda_{n}=\exp \left(n^{\alpha}\right)$ and $2 / 3<a<1$ then

$$
\left|a_{n}\right|^{\varepsilon}=O\left(\exp \left(\varepsilon C n^{2(1-\alpha)}-\varepsilon q^{-1} n^{\alpha}\right)\right),
$$

and this estimate shows that $\sum\left|a_{n}\right|^{\varepsilon}$ converges.

THEOREM 3.3. There exists a sequence $\left\{\lambda_{n}\right\}$ such that if $f(x)=\sum_{n=1}^{\infty} a_{n} \exp \left(-\lambda_{n} x\right)$ converges for each $x>0$ and $f^{\prime \prime} \in L^{1}(0, \infty)$ then $\sum\left|a_{n}\right|^{\varepsilon}$ converges.

Proof. This follows from Theorem 2.4. The proof is similar to that of Theorem 3.2 .

\section{REFERENCES}

1. J. M. Anderson and K. G. Binmore, Coefficient estimates for lacunary power series and Dirichlet series. I, Proc. London Math. Soc. (3) 18 (1968), 36-48. MR 36 \#6624.

2. D. Gaier, On the coefficients and the growth of gap power series, SIAM J. Numer. Anal. 3 (1966), 248-265. MR 34 \#4492.

3. G. Halász, Remarks to a paper of D. Gaier on gap theorems, Acta Sci. Math. Szeged 28 (1967), 311-322. MR 36 \#4199.

4. K. Hoffman, Banach spaces of analytic functions, Prentice-Hall Series in Modern Analysis, Prentice-Hall, Englewood Cliffs, N. J., 1962. MR 24 \#A2844. 
5. S. Mandelbrojt, Séries lacunaries, Paris, 1936.

6. - Séries adhérentes, régularisation des suites, applications, Gauthier-Villars, Paris, 1952. MR 14, 542.

7. W. Rudin, A converse to the high indices theorem, Proc. Amer. Math. Soc. 17 (1966), 434-435. MR 32 \#6098.

8. L. Schwartz, Étude des sommes d'exponentielles réeles, Actualités Sci. Indust., no. 959, Hermann, Paris, 1943. MR 7, 294.

9. F. Sunyer Balaguer, Approximation of functions by sums of exponentials, Collect. Math. 5 (1952), 241-267. (Spanish) MR 15, 694.

10. - Approximation of functions by linear combinations of exponentials, Collect. Math. 17 (1965), 145-177. (Spanish) MR 34 \#6413.

11. E. C. Titchmarsh, Introduction to the theory of Fourier integrals, 2nd ed., Clarendon Press, Oxford, 1948.

12. - The theory of functions, Oxford Univ. Press, Oxford, 1952.

\author{
Michigan State University, \\ East Lansing, Michigan 48823 \\ WESTFIELD COLLEGE, UNIVERSITY OF LONDON, \\ LONDON, ENGLAND
}

